Somnologie $2021 \cdot 25: 253-254$

https://doi.org/10.1007/s11818-021-00339-z Angenommen: 8. November 2021

(c) The Author(s), under exclusive licence to Springer Medizin Verlag GmbH, ein Teil von Springer Nature 2021

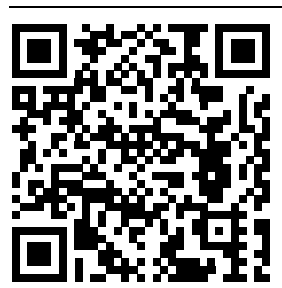

QR-Code scannen \& Beitrag online lesen

\title{
Schwangerschaft und Schlafstörungen
}

\author{
Maritta Orth ${ }^{1} \cdot$ Kurt Rasche ${ }^{2}$ \\ ${ }^{1}$ Medizinische Klinik III, Pneumologie, Pneumologische Onkologie, Allergologie, Schlaf- und \\ Beatmungsmedizin, Theresienkrankenhaus und St. Hedwig Klinik GmbH, Mannheim, Deutschland \\ ${ }^{2}$ Klinik für Pneumologie, Allergologie, Schlaf- und Beatmungsmedizin, Bergisches Lungenzentrum, \\ Wuppertal, Deutschland
}

Die Schwangerschaft stellt formal einen Normalzustand in der fertilen Phase der Frau dar. Dennoch kann dieser „physiologische Zustand" durch endogene und exogene Faktoren zu einer Bedrohung für Mutter und Fötus werden. Während 1870 die Säuglingssterblichkeit noch 250 auf 1000 Geburten betrug, sind es heute noch 3/1000 Geburten, die innerhalb des ersten Lebensjahres versterben (Bundesinstitut für Bevölkerungsforschung).

Ein entscheidender Meilenstein war hierbei sicherlich die 1849 von Ignaz Semmelweis eingeführte Händedesinfektion bei Hebammen und Geburtshelfern. Weitere Maßnahmen zur Sicherung der Gesundheit von Schwangeren und Föten wurden im Verlauf des vergangenen Jahrhunderts eingeführt und immer mehr verfeinert.

Ein sicherlich immer noch unterschätztes Schwangerschaftsrisiko sind die Veränderungen der Atmung im Schlaf bei der Mutter. Die physiologischen Veränderungen (z.B. bessere Durchblutung der Schleimhäute des oberen Atmungstraktes, hochstehendes Zwerchfell infolge der zunehmenden Uterusgröße) können zu schlafbezogenen Atmungsstörungen (Schnarchen bis hin zur obstruktiven Schlafapnoe) führen, die sowohl die Schwangere als auch den Fötus gefährden (Eklampsie, Präeklampsie, Diabetes mellitus, erhöhte Müttersterblichkeit, längere Hospitalisierungsraten, Entwicklungsretardierung, Fehl- oder Frühgeburtlichkeit bis hin zu sog. „still births"). Hierbei sind besonders die Schwangeren gefährdet, bei denen schon vor Eintritt der Schwangerschaft Übergewicht bestand. Mit der CPAP (continuous positive airway pressure)-Therapie steht heute eine effiziente Therapie zur Vermeidung, zumindest jedoch zur Verminderung der sog. „adverse pregnancy-outcomes" zur Verfügung.

Andere Schlafstörungen oder schlafassozierte Störungen können ebenfalls in der Schwangerschaft eine Bedeutung haben, so das Restless Legs Syndrom, welches in der Schwangerschaft gehäuft auftritt und den erholsamen Schlaf der Schwangeren zusätzlich mindert. Die Diagnosestellung erfolgt exakt nach den allgemein etablierten diagnostischen Kriterien und 


\section{Editorial}

unter Ausschluss anderer Faktoren, die eine RLS-Symptomatik vortäuschen können.

Im Hinblick auf die Betreuung von Schwangeren, die schon vor Eintreten der Schwangerschaft unter einer Hypersomnie und hierbei insbesondere einer Narkolepsie leiden, muss eine exakte Aufklärung der Eltern, bestenfalls vor Eintritt der Schwangerschaft, im Hinblick auf die Fertilitätsrisiken sowie zu erwartende Komplikationen der entsprechenden Medikamente stattfinden. Gerade in diesen Fällen müssen Neurologen, Gynäkologen aber auch Hausärzte in einer engen Kooperation stehen.

Somit stellt zwar die Schwangerschaft einen „natürlichen Zustand “ dar. Im Verlauf können aber primär physiologische Veränderungen zu Komplikationen führen, die nicht nur unangenehm sein können, sondern sowohl für Mutter als auch den Föten ein erhebliches Risiko darstellen können. Das vorliegende Heft soll daher darstellen, dass es sich bei der Schwangerschaft um ein multifaktorielles Geschehen handelt, welches ein hohes Maß an Interprofessionalität erfordert.

\section{Korrespondenzadresse}

\section{Prof. Dr. med. Maritta Orth}

Medizinische Klinik III, Pneumologie, Pneumologische Onkologie, Allergologie, Schlaf- und Beatmungsmedizin, Theresienkrankenhaus und St. Hedwig Klinik GmbH

Bassermannstraße 1, 68165 Mannheim,

Deutschland

m.orth@theresienkrankenhaus.de

Interessenkonflikt. M. Orth und K. Rasche geben an, dass kein Interessenkonflikt besteht.

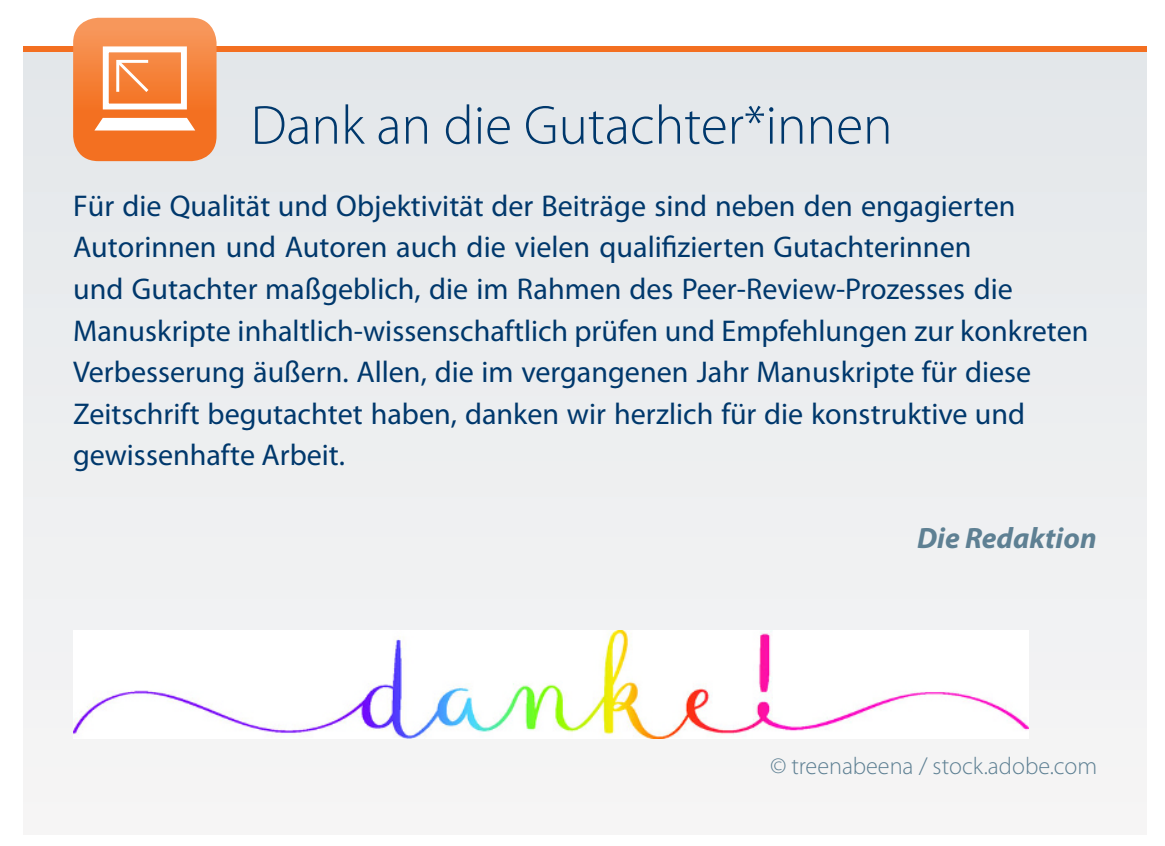

